Case Report

\title{
Classic Testicular Seminoma in Men Aged 50 Years or Over: A Case Report and Review of the Literature
}

\author{
Rawad Abou Zahr (D), ${ }^{1}$ Khalil Chalhoub ${ }^{1}$, ${ }^{1}$ Imad Matta, ${ }^{1}$ Sawsan Horani, ${ }^{2}$ \\ Zarouhie Bedoyan, ${ }^{3}$ Georges El Hachem, ${ }^{4}$ Imad Ghantous $\mathbb{D}^{\mathbb{D}},{ }^{1}$ and Michel Jabbour $\mathbb{D}^{1}$ \\ ${ }^{1}$ Department of Urology, University of Balamand, Saint George Hospital University Medical Center, Beirut 1100 2807, Lebanon \\ ${ }^{2}$ University of Balamand, Faculty of Medicine and Medical Sciences, Beirut 1100 2807, Lebanon \\ ${ }^{3}$ Department of Pathology, University of Balamand, Saint George Hospital University Medical Center, Beirut 1100 2807, Lebanon \\ ${ }^{4}$ Department of Hematology-Oncology, University of Balamand, Saint George Hospital University Medical Center, \\ Beirut 1100 2807, Lebanon
}

Correspondence should be addressed to Michel Jabbour; drmjabbour@gmail.com

Received 10 June 2019; Revised 10 August 2019; Accepted 26 September 2019; Published 24 November 2019

Academic Editor: David Duchene

Copyright ( 2019 Rawad Abou Zahr et al. This is an open access article distributed under the Creative Commons Attribution License, which permits unrestricted use, distribution, and reproduction in any medium, provided the original work is properly cited.

\begin{abstract}
Testicular cancer is the most frequent solid tumor detected in young adult men. Germ cell tumors (GCTs), particularly seminomas, are the most common type of testicular neoplasms seen in that age population. Most publications have reported decreasing incidence of GCTs in patients above forty years of age. Since the biologic activity of seminomas appears similar across ages, recommended management of senior adults involves a multimodal therapy of radical inguinal orchiectomy with radiation or cytotoxic treatment as needed. Attenuating chemotherapy dosages are critical to ensure better tolerability of associated adverse events. Here we report a case series of 2 men older than fifty years of age with metastatic testicular seminoma. We aim to emphasize a rare clinical entity encountered in the senior adult population.
\end{abstract}

\section{Introduction}

Testicular GCTs account for most testicular cancers in young adult men and have a peak incidence in the second and third decades of life $[1,2]$. Of all reported testicular cancers in the United States, more than $95 \%$ are predominantly GCTs, half of which are seminomas [2,3]. Globally, seminoma incidence rates were highest in European and Northern American countries and lowest in Asian and African countries [4]. The highest incidence rates were witnessed in Norway and Denmark (5.5/100,000 man-years), and the lowest rates were encountered in India and Uganda $(<0.5 / 100,000$ man-years) [4]. The World Health Organization's (WHO) 2016 updated classification of testicular GCTs now categorizes them based on histopathology into seminomas, non-seminomas, and spermatocytic tumors [5].

Despite their high incidence in young adults, testicular GCTs are rarely encountered in senior adults. Only less than $4 \%$ of patients with testicular GCTs are above sixty five years of age [6]. Recent epidemiologic studies highlight an increased incidence of seminomas across all age groups as well as an older age at diagnosis [7]. Mean age of diagnosis has shifted from 34 to 39 years for seminomas, as opposed to 26 to 31 years for non-seminomas [7]. In most clinicopathological studies seminomas, non-seminomas, and spermatocytic tumors account for $50-55 \%, 45-50 \%$ and $1 \%$ of testicular cancers, respectively $[7,8]$. The biological phenotype and malignant potential remain the same across age groups suggesting that similar treatment regimens should be pursued [9].

Macroscopically, seminomas are well circumscribed, tan to pale yellow lesions with necrotic or hemorrhagic foci. The cells are arranged into nests and sheets with intercepting thin fibrovascular septa which have lymphocytes and sometimes syncytiotrophoblasts.

Cells have clear or eosinophilic cytoplasm with large nuclei and prominent nucleoli and they are immunoreactive to SALL4, OCT3/4, C-KIT, D2-40, and SOX17 [9]. Patients with seminomas can have elevated $\beta$-hCG and LDH but never AFP $[8,10]$. Only about $30 \%$ of patients can have elevated $\beta$-hCG. LDH is a positive non-specific marker that reflect tumor burden [10].

Common metastatic sites of testicular seminomas involve the lungs and the liver. Rarely, it can also metastasize to the bones and the brain [11]. Even at advanced stages, testicular 


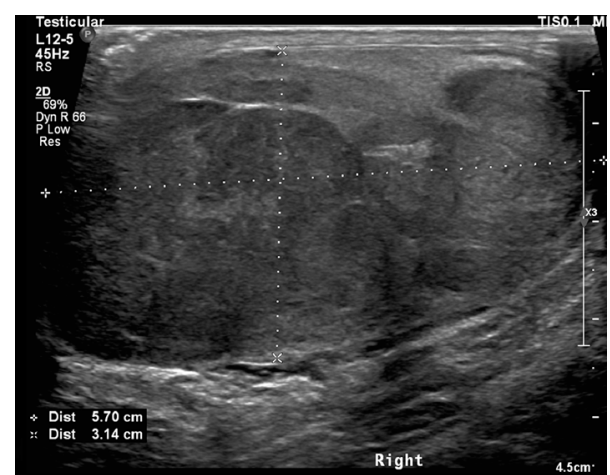

FIGURE 1: Ultrasound of right testicle.

seminomas can still have high cure rates. The most common stage of presentation, stage I seminoma, has a cure rate above $95 \%$ [12]. Considering all stages, the survival rate is $86 \%$ and $71 \%$ for the 5- and 10-year survival rate, respectively [9]. Important predictors of metastasis involve lymphovascular invasion by the primary tumor while predictors of relapse involve tumor size $(>4 \mathrm{~cm})$ and invasion of the rete testis. When both relapse predictors are absent, there is only a $6 \%$ risk of recurrence $[12,13]$.

\section{Case 1}

A 56-year-old male was presented to our facility for testicular pain of 3 weeks duration associated with progressive increase in the size and swelling of the right testicle. Physical examination revealed enlarged and rigid right testicle associated with tenderness.

Ultrasonography of the testicles revealed a hypoechoic heterogeneous well circumscribed lesion measuring more than $5.7 \times 3.1 \times 4.1 \mathrm{~cm}$ with nodularity and vascularity (Figure 1 ).

Metastatic work up included the Chest Abdomen Pelvis CT scan which revealed multiple enlarged retroperitoneal, para-aortic, and aorto caval lymphadenopathies. The largest lymph node was found in the left para aortic space with size $3 \times 5 \mathrm{~cm}$. Tumor markers were done upon admission showing negative AFP $(2.31 \mathrm{ng} / \mathrm{ml})$, slightly elevated BHCG (9.91 mIU/ $\mathrm{ml}$ ) and increased LDH levels $(801 \mathrm{U} / \mathrm{L})$. The patient underwent a right radical inguinal orchiectomy. The gross histology study demonstrated parenchyma of the testes occupied by a tan yellow, more or less well demarcated soft tumor mass measuring $5 \times 2 \times 2 \mathrm{~cm}$ with areas of yellow necrosis. Diagnosis concluded classic seminoma stage pT2, with lymphovascular invasion and negative spermatic cord margins. No evidence of invasion of the rete testis, tunica albuginea, tunica vaginalis, or epididymis. Immunostaining revealed positive expression of PLAP and SALL4 antibodies by tumor cells and negative anti-cytokeratin antibody.

Patient was diagnosed with stage IIB classical seminoma, he was started on cytotoxic chemotherapy consisting of 1 cycle of BEP protocol (Bleomycin, Etoposide, and Cisplatin) followed by 3 cycles of EP (Etoposide and Cisplatin) without Bleomycin because of pulmonary toxicity. A spirometry was performed since the patient was at high risk for pulmonary

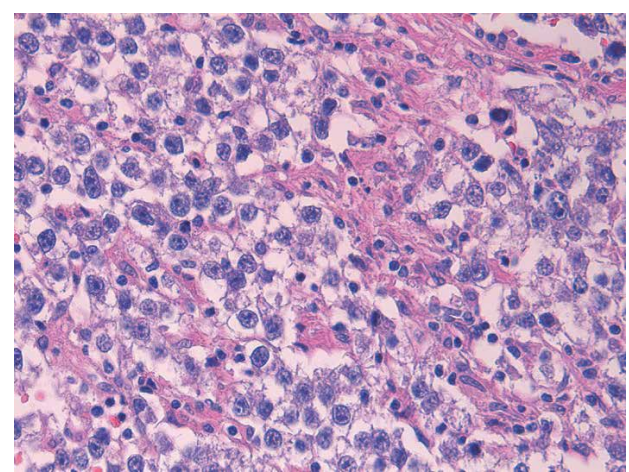

(a)

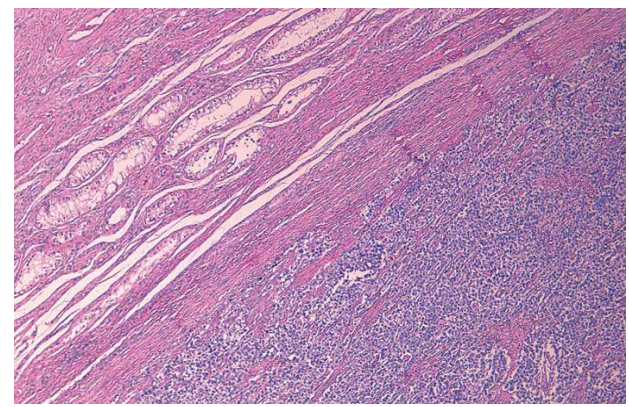

(b)



(c)

FIGURE 2: (a): High-power view shows round to polyhedral cells with distinct cell borders, and sparse lymphocytic infiltrate within fibrous septa, (b): Low-power view of the tumor (right) with background intratubular germ cell neoplasia (upper left), (c): Medium-power view of the tumor shows sheets of uniform clear cells divided into poorly demarcated lobules by delicate fibrous septa.

complications secondary to his smoking status and showed a 25\% decrease in DLCO (Diffusing capacity of the Lungs for Carbon Monoxide), prompting bleomycin interruption [14]. He received 5 days of granulocytes colony stimulating factors (G-CSF) to prevent febrile neutropenia. The LDH normalized at the end of the first cycle. The Chest Abdomen Pelvis CT scan was repeated after the fourth cycle revealing an excellent partial response with persistence of 2 lymph nodes of 1.8 and $1.9 \mathrm{~cm}$, with necrotic centers. The 4 cycles of chemotherapy were smooth without any renal, oto- or neuro-toxicity. He did not have any episode of febrile neutropenia. The DLCO re-increased by $15 \%$, without reaching the pre-chemotherapy value. Since the remaining lymph nodes were less than $3 \mathrm{~cm}$, close follow up with CT scan was 


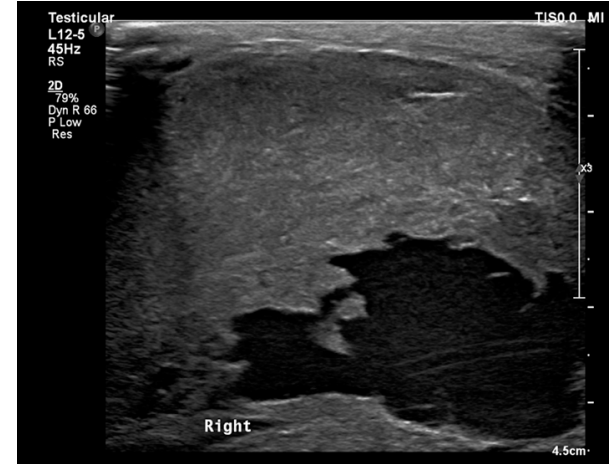

FIgURE 3: Ultrasound of right testicle.

scheduled on a bi-yearly basis for the first year and then yearly once for five years. To date the patient is being followed up 24 months after diagnosis and is doing well with no evidence of recurrence.

\section{Case 2}

A 65-year-old male patient, previously healthy, presented to our emergency department for a new onset of testicular pain following by swelling of his right testicle that started 4 weeks ago.

On physical examination, the patient had an apparent large and rigid right testicle with moderate tenderness upon palpation.

Ultrasonography of the testicles revealed a very large heterogenous vascularized mass in the right scrotum showing cystic components and containing fine echoes (Figure 3 ). The patient was admitted for further diagnosis and management. On the ward, tumor markers were done showing normal AFP and BHCG levels, $1.65 \mathrm{ng} / \mathrm{ml}$ and $3.63 \mathrm{mIU} / \mathrm{ml}$ respectively, and an elevated LDH level of $891 \mathrm{U} / \mathrm{L}$.

The Chest Abdomen Pelvis CT scan was done as metastatic workup and showed few well circumscribed subpleural solid lung nodules measuring $1.4 \mathrm{~cm}$ in the apical segment of the right upper lobe, $2.5 \times 2.8 \mathrm{~cm}$ in the anterior basal segment of right lower lobe and $3.3 \times 1.6 \mathrm{~cm}$ in the posterior segment of the left lower lobe. Bulky necrotic retroperitoneal adenopathy was also noted in the portacaval region measuring up to $3.5 \times 3 \times 3 \mathrm{~cm}$ consistent with nodal metastases. After confirming the diagnosis, the patient underwent right radical orchiectomy. The final pathology came out as Classic Pure Seminoma, measuring $13 \mathrm{~cm}$ in largest dimension with extensive areas of necrosis and cystic degeneration. The Tumor focally invaded the tunica albuginea and reached the surface of the inked visceral layer of the tunica vaginalis. Invasion of the spermatic cord was also noted. Resection margin of the spermatic cord was free on neoplasia. Final diagnosis established a Classic Pure Seminoma stage pT3 N2 M1a.

The patient was classified to have good prognosis according to the International Germ Cell Cancer Collaborative Group (IGCCCG) risk classification and was started on 3 cycles of BEP protocol. His chemotherapy sessions were uneventful and smooth with no reported complications. A similar protocol of follow up with the Chest Abdomen Pelvis CT scan was scheduled on a bi-yearly basis for the first year and then once yearly for five years. To date, the patient is doing well 14 months post diagnosis and is currently being followed up as per the assigned protocol.

\section{Discussion}

Testicular GCTs is the number one cancer detected in young male adults, accounting for $21 \%$ of diagnosed invasive cancers in patients between 20 and 40 years of age. GCTs are rarely encountered in prepubertal males as well as senior adult men [15]. Most articles reviewed in the literature report showed declining incidence of testicular GCTs beyond the age of 40 $[15,16]$.

Histologically, studies showed that seminomas predominate in patients above fifty years old, while non-seminomas predominate in younger counterparts [16].

The clinical characteristics of seminomas in the elderly has had conflicting findings in the literature. The most common presenting symptom in older adults as well as young adults is a palpable, and painless scrotal mass that can be associated with discomfort [8]. Similarly, our patients were presented to our facility for a right testicular mass. According to Berney et al., seminomas in patients aged 50 and older tend to present at advanced stages and demonstrate aggressive behavior. Fifty cases were reviewed, and the results show that in senior adults with GCTs have a significantly larger macroscopic size and more frequent invasion of the rete testis and vascular invasion, as well as less frequent intratubular germ cell neoplasia [16].

One of the earliest publications on seminomas defines them as malignant GCTs that arise from the germinal epithelium of testicles and originate from precursor cells of the spermatogonium [17]. Rarely, however; about 5\% of primary seminomas can also arise in extragonadal sites such as the pineal gland of the brain, the anterior mediastinum, and the retroperitoneum [18]. Primary GCTs in senior adults most commonly arise in the mediastinum followed by the retroperitoneum, and the CNS, while primary GCTs in young adult men are most commonly detected in the cranium [18].

A recent large-scale study by the Cancer Registration Committee of the Japanese Urological Association (2016) states that only $11 \%$ (123 out of 1119) of patients above 50 years old developed primary GCTs and $74.8 \%$ (91) of the GCTs in that age category were seminomas. $79.5 \%$ of the seminomas were stage I, $6.6 \%$ were stage II, and $13.9 \%$ were stage III according to the Union for International Cancer Control (UICC) classification. Furthermore, $84 \%$ of reported metastatic GCTs had a good prognosis as per the International Germ Cell Cancer Collaborative Group (IGCCC) vs. $16 \%$ and $0 \%$ had intermediate and poor prognosis, respectively. Survival, in months, included 72, 20 and 0 patients at 24, 48, and 60 months, respectively [19].

Wheater et al. studied 60 patients aged 60 and above. 44 patients were reported to have pure seminoma (73\%) while the remaining 16 had non seminomatous and mixed tumors. Among the 44 patients with seminomas, 33 patients had stage I seminoma, 2 had stage II seminoma, and the rest had 
stage III seminoma. The median survival was 194 months for those with stage I seminoma and 70 months with metastatic disease [20].

In a case report written by Foell et al., an 86-year-old, the oldest patient in literature, with classic seminoma was compared with 4 patients under the age of 50. The authors state that the histologic features, biologic activity, and phenotype of the seminoma were similar and suggested following similar treatment algorithms [9]. The primary therapy for testicular seminoma is radical inguinal orchiectomy as it allows for accurate staging and histologic diagnosis of the tumor. Scrotal violations are avoided at all cost as they increased the risk of seeding of the scrotum and damaging regional lymphatic drainage [2].

Adjuvant therapy depends on staging but usually involves a multimodal approach. For stage I seminomas, active surveillance or carboplatin are recommended. Stage IIA and IIB seminomas are managed with dog-leg radiotherapy; the irradiation of the para-aortic lymph nodes with the ipsilateral iliac lymph nodes. Chemotherapy including 4 cycles of EP protocol (etoposide and cisplatin) or 3 cycles of BEP protocol (bleomycin, etoposide, and cisplatin), are used for stage IIA, IIB, IIC as an alternative to radiation as well as stage III seminomas [21]. For stage II seminomas, both radiotherapy and cisplatin-based chemotherapy are reported to be equally effective with the latter favored due to lower relapse rates. To note, carboplatin monotherapy has also been tried in stage II seminomas with reports of worse outcomes, however; when combined with radiotherapy it has been proven promising [21].

Testicular GCTs are highly responsive to chemotherapy and radiotherapy, thus; making a good outcome highly likely [16]. Both modalities, however, carry risks. Radiation runs the risk of secondary malignancies and cardiotoxicity, while the BEP protocol can cause cardiopulmonary toxicity, neurotoxicity, nephrotoxicity, and infertility as well as secondary cancer $[3,22]$. Our patient was managed by a right radical inguinal orchiectomy and completed 1 cycle of the BEP protocol but was later switched to 3 cycles of EP as he developed a pulmonary reaction to Bleomycin at the start of his second cycle.

Most recent studies recommend following identical treatment algorithms, owing to the similar phenotype of seminomas in both age populations, but it is important to discuss factors hypothesized to lower treatment outcomes in senior adults. The age-related functional decline or associated comorbidities seen in this age bracket renders them less tolerable to the adverse effects and toxicities associated with chemotherapy. Prophylactic attenuation of chemotherapy dosage in senior adults is essential [23].

Those with contraindications to Bleomycin can alternatively receive 4 cycles of EP if they are in the good prognosis group and 4 cycles of VIP if they are in the intermediate or poor prognosis groups. The alternative EP and VIP regimens have been reported to have equivalent success rates, though the former regimen has been associated with lower rates of disease free survival $[20,22]$. Wheater et al. modified the conventional BEP regimen by delivering a single empiricallyreduced dose of 30,000 International Units of Bleomycin per cycle on the second day, without evidence of significant pulmonary toxicity [20]. To note, other trials have attempted to reduce Etoposide from the conventional dose of $500 \mathrm{mg} / \mathrm{m}^{2}$ to $360 \mathrm{mg} / \mathrm{m}^{2}$ with complete exclusion of Bleomycin but reported poorer outcomes [20].

For instance, advanced age and associated declining renal function in senior patients pose a $5 \%$ risk of Bleomycin pulmonary toxicity due to advanced age alone and a $20 \%$ risk when pooled with impaired renal function [19]. Thus, cautious selection of chemotherapeutic agents as well as dosages is necessary. According to Beyer et al., the BEP protocol is the mainstay of chemotherapy for senior patients with metastatic disease in good, intermediate, and poor prognosis groups [22].

Another concern is chemotherapy-induced nephrotoxicity particularly associated with cisplatin. The primary measures of mitigating cisplatin-related nephrotoxicity involve the combination of adequate hydration ( $\geq 3 \mathrm{~L} /$ day) and osmotic diuresis with mannitol during chemotherapy. Alternatively, patients with declining renal function could substitute cisplatin with carboplatin. Carboplatin dose setting based on renal function after target AUC has been established is also recommended [24].

The optimal salvage therapy for elderly patients with seminomas who relapse after surveillance, adjuvant carboplatin or adjuvant radiotherapy remains unknown. Several combinations of chemotherapy were used as a first-line salvage treatment, including conventional dose chemotherapy (CDCT) and high dose chemotherapy (HDCT). HDCT was reserved for patients with unfavorable prognostic factors and high risk of failure of salvage therapy. CDCT regimens included 4 cycles of VIP, TIP or VeIP, and HDCT which comprised of 2-3 sequential cycles of etoposide and carboplatin. In the second and third salvage therapy setting, other agents such as oxaliplatin, gemcitabine, and paclitaxel have been recommended as single agent or combination chemotherapy [21, 22].

If residual seminoma masses present post chemotherapy or radiotherapy, close monitoring with tumor serum markers and imaging is recommended, especially if residual masses are $>3 \mathrm{~cm}$. The Fluorodeoxyglucose (FDG)-PET scanning is a reliable imaging modality with high prognostic value for residual masses post treatment. Patients with positive FDG-PET scans for residual masses should get biopsied. Based on histology, further treatment options could include observation, chemotherapy, radiation or surgical resection [25].

Follow up protocols vary based on staging and consist of active surveillance, tumor markers, and imaging (the chest abdominopelvic CT, the brain CT and CXR) [12]. Testicular ultrasound is reserved for ambiguous exams [26]. For stage I seminoma, in the first 2 years post-surgery, tumor markers test and physical exam should be conducted every 4 months, and abdominopelvic CT and CXR every 6 months. In years $3-5$, the physical exam and tumor markers test are to be done once per year and abdominopelvic CT on the third and fifth year. For metastatic seminoma, in the first 2 years, tumor markers test, physical exam, and CXR are to be done every 3 months, abdominopelvic CT every 6 months, and the brain and the chest CT every 12 months. For years 3-5, tumor markers, physical exam, and CXR should be done every 6 years, and the chest abdominopelvic CT and the brain CT every 12 months [12]. 


\section{Conclusion}

Testicular GCTs are neoplasms encountered frequently in young adults and are rarely seen in the senior adult population. Histologically, seminomas predominate in patients over fifty years old and have retained the phenotype, biologic activity, and malignant potential. Seminomas remain curable and have good prognosis in senior adults even at advanced stages. Treatment regimens similar to younger patients can be employed as long as chemotherapy dosages are empirically reduced, and precautions are taken to mitigate chemotherapy-related toxicities.

\section{Conflicts of Interest}

The authors declare that they have no conflicts of interest.

\section{References}

[1] G. E. Hachem, S. Sideris, and T. Gil, "56 years-old, an old age for pure seminomatous germ cell tumor: Case report and review of the literature," Cancer Reports and Reviews, vol. 2, no. 1, pp. 1-4, 2017.

[2] N. Boujelbene, A. Cosinschi, N. Boujelbene et al., "Pure seminoma: a review and update," Radiation Oncology, vol. 6, no. 1, p. 90, 2011.

[3] A. A. Ghazarian, B. Trabert, S. S. Devesa, and K. A. McGlynn, "Recent trends in the incidence of testicular germ cell tumors in the United States," Andrology, vol. 3, no. 1, pp. 13-18, 2014.

[4] B. Trabert, J. Chen, S. S. Devesa, F. Bray, and K. A. Mcglynn, "International patterns and trends in testicular cancer incidence, overall and by histologic subtype, 1973-2007," Andrology, vol. 3, no. 1, pp. 4-12, 2014.

[5] S. R. Williamson, B. Delahunt, C. Magi-Galluzzi et al., "The World Health Organization 2016 classification of testicular germ cell tumours: a review and update from the International Society of Urological Pathology Testis Consultation Panel," Histopathology, vol. 70, no. 3, pp. 335-346, 2016.

[6] N. Ismaili, S. Naciri, S. Afqir et al., "A rare case of advanced testicular seminoma in a 78-year-old man managed successfully with carboplatin based chemotherapy: a case report," Cases Journal, vol. 1, no. 1, p. 357, 2008.

[7] C. G. Ruf, H. Isbarn, W. Wagner, M. Fisch, C. Matthies, and K. Dieckmann, "Changes in epidemiologic features of testicular germ cell cancer: age at diagnosis and relative frequency of seminoma are constantly and significantly increasing," Urologic Oncology: Seminars and Original Investigations, vol. 32, no. 1, pp. 33.e1-33.e6, 2014.

[8] J.Marko, D. J. Wolfman, A. L. Aubin, and I. A. Sesterhenn, "Testicular seminoma and its mimics: from the radiologic pathology archives," RadioGraphics, vol. 37, no. 4, pp. 1085-1098, 2017.

[9] K. Foell, M. Martens, and J. I. Izawa, "A rare case of classic testicular seminoma in an 86-year-old shows similar proliferation rate as in younger men," Urology, vol. 70, no. 5, pp. 1007.e7-1007.e9, 2007.

[10] C. G. Ruf, "Follow-up for testicular," in Cancer Urologic Oncology, A. Merseburger and M. Burger, Eds., pp. 1-11, Springer, Cham, 2019.

[11] S. Degirmencioglu and B. Degirmencioglu, "Isolated femoral muscle and bone metastases rarely encountered in testicular seminoma," Case Reports in Oncological Medicine, vol. 2013, Article ID 780493, 3 pages, 2013.

[12] P. Albers, W. Albrecht, F. Algaba et al., "Guidelines on testicular cancer: 2015 update," European Urology, vol. 68, no. 6, pp. 10541068, 2015.

[13] P. Warde, M. Gospodarowicz, D. Banerjee et al., "Prognostic factors for relapse in stage I testicular seminoma treated with surveillance," Journal of Urology, vol. 157, no. 5, pp. 1705-1710, 1997.

[14] J. Lauritsen, M. Kier, M. Bandak et al., "Pulmonary function in patients with germ cell cancer treated with bleomycin, etoposide, and cisplatin," Journal Of Clinical Oncology, vol. 34, no. 13, pp. 1492-1499, 2016.

[15] B. Hayes-Lattin and C. R. Nichols, "Testicular cancer: a prototypic tumor of young adults," Seminars in Oncology, vol. 36, no. 5, pp. 432-438, 2009.

[16] D. M. Berney, A. Y. Warren, M. Verma et al., "Malignant germ cell tumours in the elderly: a histopathological review of 50 cases in men aged 60 years or over," Modern Pathology, vol. 21, no. 1, pp. 54-59, 2007.

[17] J. Innes, W. F. Harvey, and E. K. Dawson, "Debatable tumors in human and animal pathology-iii," Seminoma. Edinburgh Medical Journal, vol. 45, no. 1, pp. 36-42, 1938.

[18] A. B. Shinagare, J. P. Jagannathan, N. H. Ramaiya, M. N. Hall, and A. D. Van den Abbeele, "Adult extragonadal germ cell tumors," American Journal of Roentgenology, vol. 195, no. 4, pp. W274-W280, 2010.

[19] T. Kawai and Y. Tanaka, "Clinical characteristics of testicular germ cell tumors in patients aged 50 years and older: a largescale study from the cancer registration committee of the japanese urological association," International Journal of Urology, vol. 24, no. 2, pp. 124-128, 2016.

[20] M. J. Wheater, J. Manners, L. Nolan, P. D. Simmonds, M. C. Hayes, and G. M. Mead, "The clinical features and management of testicular germ cell tumours in patients aged 60 years and older," BJU International, vol. 108, no. 11, pp. 1794-1799, 2011.

[21] F. Honecker, J. Aparicio, D. Berney et al., "ESMO consensus conference on testicular germ cell cancer: diagnosis, treatment and follow-up," Annals of Oncology, vol. 29, no. 8, pp. 16581686, 2018.

[22] J. Beyer, P. Albers, R. Altena et al., "Maintaining success, reducing treatment burden, focusing on survivorship: highlights from the third European consensus conference on diagnosis and treatment of germ-cell cancer," Annals of Oncology, vol. 24, no. 4, pp. 878-888, 2013.

[23] J. Spermon, J. Witjes, and L. Kiemeney, "Difference in stage and morphology-adjusted survival between young and elderly patients with a testicular germ cell tumor," Urology, vol. 60, no. 5, pp. 889-893, 2002.

[24] S. Horie, M. Oya, M. Nangaku et al., "Guidelines for treatment of renal injury during cancer chemotherapy 2016," Clinical and Experimental Nephrology, vol. 22, no. 1, pp. 210-244, 2018.

[25] S. Krege, J. Beyer, R. Souchon et al., "European consensus conference on diagnosis and treatment of germ cell cancer: a report of the second meeting of the european germ cell cancer consensus group: part II," European Urology, vol. 53, no. 3, pp. 497-513, 2008.

[26] K. Sachdeva, “Testicular cancer follow-up," 2018, https:// emedicine.medscape.com/article/279007-followup. 


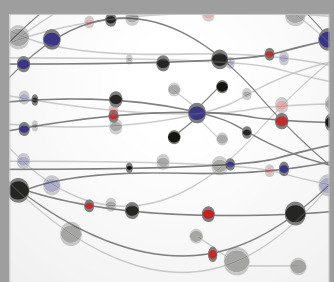

The Scientific World Journal




Gastroenterology Research and Practice

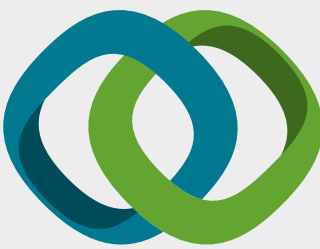

\section{Hindawi}

Submit your manuscripts at

www.hindawi.com
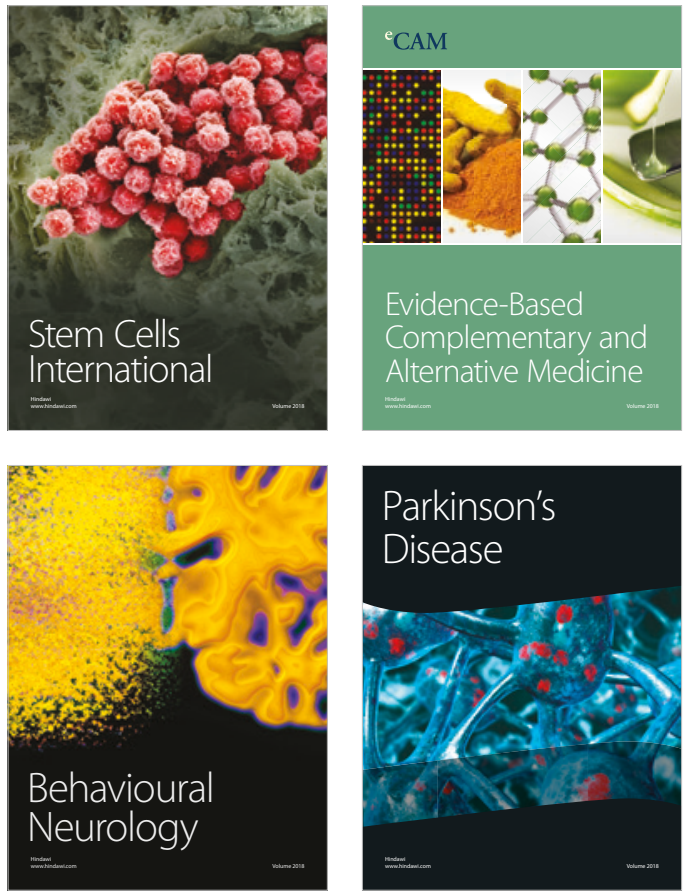

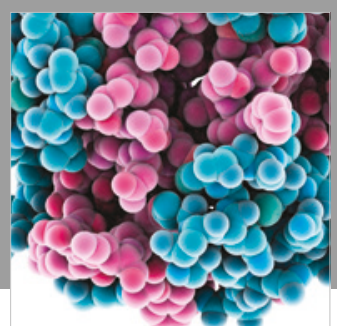

ournal of

Diabetes Research

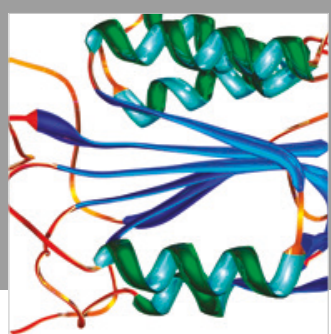

Disease Markers
